\title{
Biochemical Analysis of the Role of Cytoplasmic Ribosomes of Coprinus cinereus in Cycloheximide Resistance
}

\author{
By JOHN D. TRAYNOR, IFFAT SARDHARWALLA $\dagger$ AND \\ JANE NORTH* \\ Department of Biological Sciences, City of London Polytechnic, Calcutta House, \\ Old Castle Street, London E1 7NT, UK
}

(Received 31 July 1985; revised 15 October 1985)

\begin{abstract}
The development of an optimized in vitro polyuridylic acid-dependent polyphenylalaninesynthesizing system using cell-free extracts of the basidiomycete fungus Coprinus cinereus is described. The in vitro assay has been used to show that cycloheximide-resistant strains CY8.2, CY9.23 and Sp98, all mutant at the $c y-2$ locus, have cytoplasmic ribosomes which are more resistant to the drug than the corresponding sensitive strains, CY8, CY9 and CY3. Cycloheximide concentrations and molar ratios of cycloheximide to ribosomes required for $50 \%$ inhibition in vitro under standard assay conditions are presented for these strains. The molar ratio required for $50 \%$ inhibition in vitro is dependent on the concentration of ribosomes in the assay.
\end{abstract}

\section{INTRODUCTION}

Cycloheximide is an antibiotic that inhibits translation on eukaryotic cytoplasmic ribosomes. Mutations altering the cycloheximide sensitivity of ribosomes have been described in Saccharomyces cerevisiae (Cooper et al., 1967), Schizosaccharomyces pombe (Ibrahim \& Coddington, 1976), Podospora anserina (Crouzet et al., 1978), Neurospora crassa (Hsu, 1963; Neuhäuser et al., 1970) and Saccharomyces fragilis (Siegel \& Sisler, 1965). A large number of mutants of Coprinus cinereus resistant to cycloheximide in vivo have been isolated after UV irradiation of oidia. These mutants belong to the $c y-2$ locus which maps on linkage group II (North, 1982; Traynor, 1983). This paper describes the development of an in vitro protein synthesis assay for $C$. cinereus and its use to show that three cycloheximide-resistant mutants have ribosomes altered in their response to the antibiotic. A preliminary report has been made (Traynor \& North, 1981).

\section{METHODS}

Materials. Chemicals for the culture medium of $C$. cinereus were obtained from either Difco or BDH. Cycloheximide (actidione) was supplied by Koch-Light, and DNA and p-aminobenzoic acid by Sigma. The buffer constituents were purchased from BDH and Sigma. The chemicals for the cell-free protein-synthesizing system were supplied by Boehringer, with the exception of spermidine (Aldrich) and polyuridylic acid [poly(U)] (Calbiochem). Uniformly labelled $\left[{ }^{14} \mathrm{C}\right]$ phenylalanine was from Amersham.

Strains and nomenclature. The strains of $C$. cinereus used are listed in Table 1. Cycloheximide-resistant mutant strains were produced by UV mutagenesis of cycloheximide-sensitive strains (North, 1982; Traynor, 1983). Mutants isolated from CY strains were numbered according to their parent strains, for example CY8.2 was derived from CY8. Recombinant progeny from the cross between CY9.23 and CY3 are referred to by the spore isolate number, e.g. Sp98 (North, 1982).

Cycloheximide resistance and sensitivity alleles are represented by $c y-2^{r}$ and $c y-2^{\circ}$ respectively; the presence of an active dominance modifier allele is represented by modcy $y^{+}$and its wild-type non-active allele by modcy ${ }^{-}$.

Culture medium. Complete culture medium was as described by Lewis \& North (1974); glucose was sterilized separately and added at $1 \%(\mathrm{w} / \mathrm{v})$ after the medium had been autoclaved.

† Present address: Department of Biochemistry, King's College London, Strand, London WC2R 2LS, UK. 
Table 1. Strains of Coprinus cinereus

\begin{tabular}{|c|c|c|c|c|c|c|}
\hline \multirow[b]{2}{*}{ Strain } & \multicolumn{2}{|c|}{ Mating type } & \multirow[b]{2}{*}{ Markers } & \multirow{2}{*}{$\begin{array}{l}\text { Cycloheximide } \\
\text { resistance } \\
\text { in vivo }\end{array}$} & \multirow[b]{2}{*}{ Origin } & \multirow[b]{2}{*}{ Reference } \\
\hline & A & B & & & & \\
\hline $\mathrm{CY} 8$ & 6 & 5 & $n i c-4$ paha-2 & $c y^{s}$ & WMR66A $\times$ TC4 & 1 \\
\hline $\mathrm{CY} 9$ & 6 & 6 & nic-4 paba-2 & $c y^{\prime \prime}$ & WMR $66 \mathrm{~A} \times \mathrm{TC} 4$ & 1 \\
\hline $\mathrm{CY} 3$ & 5 & 5 & me-5 chol-1 & $c y^{\circ}$ & SR54 $\times$ TC 4 & 1 \\
\hline CY 18 & 6 & 6 & me-5 chot-1 ost & $c y^{\prime \prime}$ & $\mathrm{ZR} 336 \times \mathrm{CY} 3$ & 1 \\
\hline CY 8.2 & 6 & 5 & $n i c-4$ paha-2 & $c y-2 r$ & CY8 & 2 \\
\hline CY 9.23 & 6 & 6 & $n i c-4$ paba-2 & $c y-2^{r} \bmod y^{+}$ & CY9 & 1 \\
\hline Sp98 & 6 & 6 & $m e-5$ & $c y-2^{r} \operatorname{modc} y^{-}$ & $\mathrm{CY} 9.23 \times \mathrm{CY} 3$ & $i$ \\
\hline
\end{tabular}

Abbreviations: $c y^{*}, c y-2 r$, sensitive, resistant to cycloheximide; modc $y^{+}$, dominance modifier of $c y-2 r ; c h o l$, choline requiring; paba, p-aminobenzoic acid requiring; me, methionine requiring; nic, nicotinic acid requiring; ost, ostrich morphology. Sp, spore isolate number.

* References: (1) North, 1982: (2) Traynor, 1983.

Preparation of cytoplasmic ribosomes. For isolation of ribosomes, mycelium was grown in liquid complete medium for 3 to $4 \mathrm{~d}$ at $37^{\circ} \mathrm{C}$ with continuous agilation. The culture was harvested in cheese cloth and washed with double-distilled water. Washed mycelium was frozen in liquid nitrogen and ground immediately with an equal weight of acid-washed sand and $1 \mathrm{ml}$ buffer $\mathrm{A} .\left(50 \mathrm{~mm}\right.$-Tris $/ \mathrm{HCl}, \mathrm{pH} 7.5$ at $4{ }^{\circ} \mathrm{C}, 10 \mathrm{~mm}$-magnesium acetate, $25 \mathrm{~mm}-\mathrm{KCl}, 5 \mathrm{~mm}-2$-mercaptoethanol and $250 \mathrm{~mm}$-sucrose; autoclaved at $116^{\circ} \mathrm{C}$ for $10 \mathrm{~min}$ ) per g mycelium. All subsequent manipulations were done at $4^{\circ} \mathrm{C}$. The resulting homogenate was centrifuged for $10 \mathrm{~min}$ at $10000 \mathrm{~g}$ to remove the sand and cellular debris. The supernatant was centrifuged for a further $20 \mathrm{~min}$ at $30000 \mathrm{~g}$ to remove mitochondria. After removal of surface lipid material, the supernatant fluid was centrifuged for $2.5 \mathrm{~h}$ at $100000 \mathrm{~g}$. The supernatant fraction was carefully removed and stored at $-70^{\circ} \mathrm{C}$ in $1 \mathrm{ml}$ amounts, which were used as a source of soluble factors in the cell-free protein-synthesizing system. The brownish opaque layer above the clear pellet was carefully removed and discarded and the ribosomal pellet was gently resuspended in a small volume of buffer A. The ribosomal preparation was clarified by centrifugation at $5000 \mathrm{~g}$ for $5 \mathrm{~min}$ and the clarified suspension was stored at $-70^{\circ} \mathrm{C}$ in $50 \mu \mathrm{l}$ amounts. Cell extracts frozen in liquid nitrogen and stored at $-70^{\circ} \mathrm{C}$ retained $80 \%$ of their polyphenylalanine synthesis activity for at least six months (Traynor, 1983).

The method described above to prepare cy toplasmic ribosomes from $C$. cinereus is similar to those used for other fungi (e.g. Crouzet et al., 1978). The ribosomes showed a single band ( $254 \mathrm{~nm}$ absorption) on sucrose density gradients for which a sedimentation coefficient of $74.4 \mathrm{~S}$ (Traynor, 1983) was calculated by the method of McEwen (1967). Cytoplasmic ribosomal protein concentration was determined by the Lowry method with bovine serum albumin as a standard

Poly $(U)$-directed polyphenylalanine synthesis. For in vitro polypeptide synthesis the reaction mixture of $100 \mu 1$ contained $1 \mathrm{~mm}$-ATP, $0.25 \mathrm{~mm}$-GTP, $0.6 \mu \mathrm{g}$ creat ne phosphokinase, $12 \mathrm{~mm}$-creatine phosphate, $15 \mathrm{~mm}$ - Tris/ $/ \mathrm{HCl}$, $\mathrm{pH} 7.5$ at $4{ }^{\circ} \mathrm{C}, 4.5 \mathrm{~mm}$-magnesium acetate, $20 \mathrm{~nm}-\mathrm{KCl}, 40 \mathrm{~mm}$-ammonium acetate, $1 \mathrm{~mm}$-spermidine, $4 \mathrm{~mm}$ dithiothreitol, $0.6 \mu \mathrm{g}$ yeast $\mathrm{tRNA}{ }^{\mathrm{Plic}}, 30 \mu \mathrm{g}$ poly( $\left.\mathrm{U}\right), 0.125 \mu \mathrm{Ci}\left[{ }^{1+} \mathrm{C}\right]$ phenylalanine $\left(0.513 \mathrm{Ci} \mathrm{mol}^{-1} ; 18.98 \mathrm{GBq}\right.$ $\mathrm{mol}^{-1}$ ), I mM-2-mercaptoethanol, $50 \mathrm{~mm}$-sucrose, and 2.0 $A_{260}$ units each of supernatant fraction and cytoplasmic ribosomes (Traynor, 1983). After incubation at $30^{\circ} \mathrm{C}$ for $1 \mathrm{~h}$ the reaction mixture was placed on ice and duplicate $40 \mu \mathrm{l}$ samples were removed from each tube and spotted onto Whatman GF/A glass-fibre filter discs which had been pre-treated by soaking in $10 \%(\mathrm{w} / \mathrm{v})$ trichloroacetic acid (TCA) for $10 \mathrm{~min}$. After drying, the filter discs were put through a series of washing steps as follow: $30 \mathrm{~min}$ in ice-cold $10 \%(\mathrm{w} / \mathrm{v})$ TCA containing $0.5 \%(\mathrm{w} / \mathrm{v})$ unlabelled L-phenylalanine; $10 \mathrm{~min}$ in $5 \%(\mathrm{w} / \mathrm{v})$ boiling TCA; $10 \mathrm{~min}$ in ice-cold $5 \%(\mathrm{w} / \mathrm{v})$ TCA and finally $10 \mathrm{~min}$ in ice-cold $90 \%(\mathrm{v} / \mathrm{v})$ ethanol. The filter discs were then dried and the radioactivity counted in scintillation cocktail $[0.8 \%(\mathrm{w} / \mathrm{v})$ 2,5-diphenyloxazole and $0.01 \%(\mathrm{w} / \mathrm{v})$ 1,4-di-2-(5-phenyloxazolyl)benzene in toluene], in a Beckman LS7500 liquid scintillation counter at an efficiency of $89 \%$. Each experiment was repeated on at least one occasion; duplicate assays, with two replicate determinations per sample, were done for each experiment. Mean results are reported; standard errors were within $5 \%$ of the mean.

\section{RESULTS}

Optimization of the constituents of the $C$. cinereus polyphenylalanine-synthesizing system

As no cell-free polypeptide-synthesizing system for $C$. cinereus or any other basidiomycete had been described, we developed a system based on those devised for several other fungal species, particularly that used by Crouzet et al. (1978) for Podospora anserina (Traynor, 1983). 


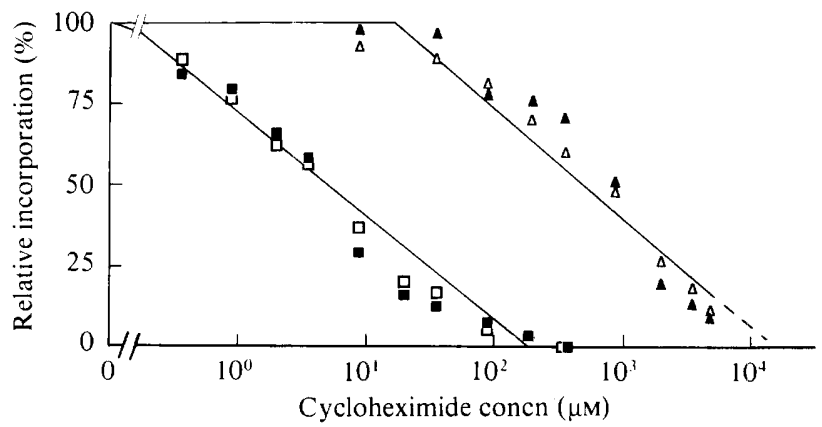

Fig. 1. Effect of varying concentrations of cycloheximide on poly(U)-directed poly $\left[{ }^{1+} \mathrm{C}\right]$ phenylalanine synthesis in reconstituted cell-free extracts from wild-type strain CY8 and cycloheximide-resistant strain CY8.2. The rates of incorporation (pmol phenylalanine incorporated $\mathrm{h}^{-1}$ per $100 \mu \mathrm{l}$ ) corresponding to $100 \%$ are shown after the respective symbol for each treatment. Homologous treatments : CY $8(\square, 36 \cdot 0)$, CY8.2 ( $\triangle, 46 \cdot 3)$; heterologous treatments: CY8 ribosomes with CY8.2 supernatant $(\boldsymbol{\square}, 46 \cdot 6), \mathrm{CY} 8.2$ ribosomes with CY8 supernatant $(\Delta, 43 \cdot 6)$.

Polyphenylalanine synthesis in vitro was totally dependent upon the presence of both cytoplasmic ribosomes and supernatant fraction. The optimum concentration ratio (in terms of $A_{260}$ ) of the two components was $1: 1$, but ratios ranging from $0.5: 1$ to $2: 1$ produced activity which was almost as high. Maximum activity was observed with $2 \cdot 0 A_{260}$ units of both fractions (Traynor, 1983). These concentrations were used in the optimized reaction mixture (see Methods).

Response of $C$. cinereus cell extracts to cycloheximide in the in vitro polyphenylalanine-synthesizing system

Investigation into the effect of cycloheximide on cell-free polyphenylalanine synthesis began with an analysis of the effects of the drug on cell extracts derived from strains CY8 and CY8.2. The growth of CY8 is sensitive to cycloheximide and that of CY8.2 is highly resistant (Traynor, 1983). Cytoplasmic ribosome-rich fractions and ribosome-free supernatant fractions from these strains were assayed in homologous and heterologous combinations (Fig. 1). Although the response curves of the sensitive and resistant strains in the homologous treatments were similar in shape, the response of the latter was displaced towards higher cycloheximide concentrations, showing that the system derived from CY8.2 was more resistant than that from CY8. The cell extracts from CY8.2 were 128 times more resistant than those from CY8 in terms of their $50 \%$ inhibitory cycloheximide concentrations and 50 times more resistant in terms of total inhibitory cycloheximide concentration.

To determine the role of the CY8.2 ribosomal and supernatant fractions in in vitro resistance to cycloheximide, heterologous combinations of CY8.2 and CY8 fractions were assayed. The dose responses of the two reciprocal combinations of ribosomal and supernatant fractions (Fig. 1) indicated that the cycloheximide concentration giving $50 \%$ inhibition was 125 times higher when the ribosomes were derived from CY8.2 than when they were derived from CY8; the response of each heterologous assay closely resembled the results obtained for the homologous assay for the strain from which the ribosomes were obtained. It is therefore inferred that the ribosomal fraction from the resistant strain CY8.2 confers resistance to cycloheximide in vitro.

\section{Analysis of the response of other $C$. cinereus strains to cycloheximide in the in vitro} polyphenylalanine-synthesizing system

To permit direct screening for cycloheximide resistance, the supernatant fraction from CY18 was used as a standard component of the assay mixture. This supernatant was chosen because it did not confer cycloheximide resistance in vitro (Table 2) whilst it produced a high polyphenylalanine-synthesizing capacity without significantly changing the response to 


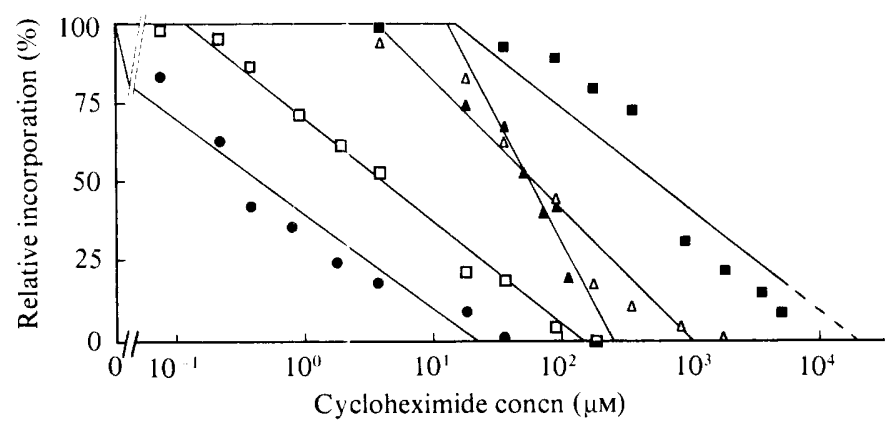

Fig. 2. Cycloheximide inhibition of $\left[{ }^{1+} \mathrm{C}\right.$ phenylalanine incorporation in a poly(U) system using cytoplasmic ribosomes from wild-type strains CY8 $(\square), C Y 9, C Y 18$ and $C Y 3(\bullet)$, and cycloheximideresistant strains $C Y 8.2(\boldsymbol{D}), C Y 9.23(\triangle)$ and $\mathrm{Sp} 98(\boldsymbol{\Delta})$. Supernatant fraction from the cycloheximidesensitive strain CY 18 was used throughout. The rates of incorporation corresponding to $100 \%$ were (pmol phenylalanine incorporated $\mathrm{h}^{-1}$ per $100 \mu \mathrm{l}$ ): CY8, 47.8; CY9, CY 18, CY3, 15.4; CY8.2, 48.5; CY9CY9.23, 18.6: Sp98, $20 \cdot 2$.

Table 2. Response to cycloheximide of (ytoplasmic ribosomes from various $C$. cinereus strains

The supernatant fraction was derived from CY $18.1 \mu \mathrm{M}$-cycloheximide per assay $\equiv 100$ pmol per assay; $2 \cdot 0 A_{260}$ units of cytoplasmic ribosomes p:r assay $\equiv 5.64$ pmol per assay (see text for details).

$\begin{array}{lcc}\begin{array}{c}\text { Source of } \\ \text { ribosomes }\end{array} & \begin{array}{c}\text { For } 50 \% \text { inhibition of polyphenylalanine synthesis } \\ \text { concn }(\mu \mathrm{M})\end{array} \\ \text { CY8 } & 4 \cdot 20 & \begin{array}{r}\text { Mol cycloheximide : } \\ \text { mol ribosomes }\end{array} \\ \text { CY9 } & (1) \cdot 45 & 85: 1 \\ \text { CY3 } & (0) 35 & 8: 1 \\ \text { CY18 } & 0 \cdot 47 & 8: 1 \\ \text { CY8.2 } & 550 & 10000: 1 \\ \text { CY9.23 } & 64 & 1100: 1 \\ \text { Sp98 } & 58 & 1030: 1\end{array}$

cycloheximide of ribosomes from any strciin compared to results obtained with homologous cellextracts (Traynor, 1983; Figs 1 and 2). The responses to cycloheximide of ribosomes from various strains are presented in Fig. 2. The response to cycloheximide in vitro was similar to that observed for the effect of this drug on the growth of the same strains (Traynor, 1983). These strains can be classified into two groups according to their $50 \%$ inhibitory concentrations in vitro: those having cycloheximide-resistant and those having cycloheximide-sensitive cytoplasmic ribosomes.

The presence of cycloheximide-resistant cytoplasmic ribosomes in CY8.2 has already been considered above. The response to cycloheximide of ribosomes from CY9.23 indicated that it too possessed cycloheximide-resistant cytoplasmic ribosomes, which were 140 times more resistant than those of the sensitive strain CY9 from which it was derived (Fig. 2, Table 2). Ribosomes from Sp98, a recombinant strain of CY9.23 and CY3, produced a similar response. However, CY8.2 ribosomes were nine times more resistant to cycloheximide than CY9.23 and Sp98 ribosomes. These three strains, CY8.2, CY9.23 and Sp98, have been shown to carry $c y-2^{\mathrm{r}}$ alleles and to be resistant to cycloheximide in vivo (North, 1982; Traynor, 1983).

Strains CY8, CY9, CY3 and CY18, shown to be cycloheximide sensitive in vivo (North, 1982; Traynor, 1983), appear also to contain r bosomes sensitive to the drug (Table 2). Strain CY8, although shown to be cycloheximide sensitive (Figs 1 and 2), was 10 times more resistant than the other three strains (Table 2). There were no significant differences between the responses of ribosomes from $\mathrm{CY} 3, \mathrm{CY} 9$ and $\mathrm{CY} 18$. 


\section{Molecular relationship between cycloheximide and the cytoplasmic ribosomes of $C$. cinereus}

In order to examine the molecular relationship between cycloheximide and the cytoplasmic ribosomes and to compare further the results obtained for various strains of $C$. cinereus, the $50 \%$ inhibitory cycloheximide concentration and cytoplasmic ribosome concentration of each strain were examined as a molar ratio (Table 2). In order to determine the number of moles of cytoplasmic ribosomes present in the in vitro assay, it was necessary to assume an $M_{\mathrm{r}}$ value for $C$. cinereus cytoplasmic ribosomes since no value has been published for this species. An $M_{\mathrm{r}}$ of 3.9 $\times 10^{6}$ was used on the assumption that the cytoplasmic ribosomes of $C$. cinereus are similar to those of other fungal species (Sissons, 1974; Berry et al., 1978). In order to calculate the concentration of the cytoplasmic ribosomes it was assumed that $1.0 A_{260}$ unit of a ribosomal preparation contained, on a weight for weight basis, the same amount each of ribosomal RNA and ribosomal protein (Nieuwenhuysen et al., 1978; Wool, 1979) and also that all the protein was cytoplasmic ribosomal protein. On these assumptions it was calculated that $9 \cdot 1 A_{260}$ units of $C$. cinereus ribosomal fraction contained $1 \mathrm{mg}$ cytoplasmic ribosomes $\mathrm{ml}^{-1}$ (Traynor, 1983). This is comparable with values of $13 A_{260}$ units for Saccharomyces cerevisiae (Sissons, 1974), $11 A_{260}$ units for Schizosaccharomyces pombe (Berry et al., 1978) and 9.3 $A_{260}$ units for Saccharomyces fragilis (Rao \& Grollman, 1967). Thus in each $100 \mu \mathrm{l}$ assay system, $2 \cdot 0 A_{260}$ units of cytoplasmic ribosomal fraction was equivalent to $22 \mu \mathrm{g}$ of cytoplasmic ribosomes of which $11 \mu \mathrm{g}$ was cytoplasmic ribosomal protein. Each assay therefore contained 5.64 pmol cytoplasmic ribosomes (Table 2).

Between six and eight molecules of cycloheximide per ribosome were necessary to produce $50 \%$ inhibition of polyphenylalanine synthesis in the cycloheximide-sensitive strains, excluding CY8 (Table 2). In contrast, in order to inhibit cytoplasmic ribosomes from CY8 to the same extent, it was necessary to have approximately 10 times the number of molecules of cycloheximide per ribosome and in the cycloheximide-resistant strains CY8.2, CY9.23 and Sp98 considerably greater numbers of cycloheximide molecules were required (Table 2). The ratios shown in Table 2 can be interpreted as a measure of the stability of the complexes formed between cycloheximide and the cytoplasmic ribosomes in the sensitive and resistant strains. There was a 10-fold difference between the response of CY8 and the other sensitive strains; however, the decrease in stability shown as a result of mutation at the $c y-2$ locus was relatively consistent (133-fold in CY8.2 and approximately 140-fold in CY9.23 and Sp98), indicating that the modifications caused by the two $c y-2^{r}$ alleles concerned were similar.

In other species, the ratio of cycloheximide to cytoplasmic ribosomes at $50 \%$ inhibition is also greater than unity: 4.8 molecules of cycloheximide were necessary for each molecule of cytoplasmic ribosomes from a cycloheximide-sensitive strain of Schizosaccharomyces pombe (Berry et al., 1978), and in Saccharomyces cerevisiae the ratios were $1.4: 1$ and $50: 1$ for cycloheximide-sensitive and cycloheximide-resistant cytoplasmic ribosomes respectively (Cooper et al., 1967).

\section{Effect of ribosome concentration on the cycloheximide response}

To study the relationship between ribosome concentration and response of polyphenylalanine synthesis to cycloheximide, ribosomes derived from the cycloheximide-resistant recombinant strain Sp98 and supernatant from CY18 (cycloheximide-sensitive) were used. The $50 \%$ inhibitory concentrations of cycloheximide decreased with increasing ribosome concentration (Table 3); Sp98 ribosomes were 1.3 times less resistant at the highest concentration than at the lowest ribosome concentration examined.

From the molecular relationships between the cytoplasmic ribosomes and cycloheximide at various concentrations of the former (Table 3), it is apparent that the number of moles of cycloheximide required for the $50 \%$ inhibition of polyphenylalanine synthesis decreased with increasing concentration of ribosomes in the assay. The standardized assay contained $2.0 A_{260}$ units of ribosomes because this produced optimum activity, while concentrations above and below this resulted in reduced polypeptide synthesis (results not presented; Traynor, 1983). The increased sensitivity to cycloheximide seen at higher ribosome concentrations may be connected with this overall inhibition; however, this does not explain the decreased sensitivity to 
Table 3. Effect of cytoplasmic ribosome concentration on inhibition of polyphenylalanine synthesis by cycloheximide

Cytoplasmic ribosomes and supernatant fractions were derived from Sp98 and CY18, respectively. $1 \mu \mathrm{M}$-cycloheximide per assay $\equiv 100 \mathrm{pmol}$ per assay. The estimated $M_{\mathrm{r}}$ of cytoplasmic ribosomes of C. cinereus is $3.9 \times 10^{6}$ (see text for details).

$\begin{array}{cc}\text { Ribosome } & 50 \% \text { inhibitory } \\ \text { concn } & \text { cycloheximide } \\ \left(A_{260} \text { units }\right) & \text { concn } \\ \text { per } 100 \mu \mathrm{l}) & (\mu \mathrm{M})\end{array}$

$\begin{array}{ll}0 \cdot 7 & 61 \\ 1 \cdot 5 & 71 \\ 2 \cdot 0 & 58 \\ 2 \cdot 5 & 42 \\ 3 \cdot 2 & 48\end{array}$

\author{
Mol cycloheximide : \\ mol ribosomes \\ for $50 \%$ inhibitory \\ cycloheximide concn
}

$\begin{aligned} & 2980: 1 \\ & 1630: 1 \\ & 1030: 1 \\ & 590: 1 \\ & 530: 1\end{aligned}$

cycloheximide at the lower ribosome concentrations. These results may best be interpreted in terms of the states in which the ribosomes may exist at different concentrations. At high concentrations ribosomes may form polysomes on individual poly(U) molecules, allowing inhibition of translation of all the ribosomes of one polysome by the binding of cycloheximide to one of them. As the poly(U) concentration in the assays was constant, polysome or higher aggregate formation was less likely at the lower ribosome concentrations, so that the overall polyphenylalanine-synthesizing system appeared more resistant at the lower concentrations.

\section{DISCUSSION}

The optimized $C$. cinereus cell-free polyphenylalanine-synthesizing system had an efficiency which compared favourably with those from other fungal species (Crouzet et al., 1978; Sissons, 1974; Berry et al., 1978). Although it is clear from Fig. 1 that the ribosomes in the cell-free assay confer cycloheximide resistance, comparison of the results for CY8 and CY8.2 ribosomes with supernatants from CY8, CY8.2 and CY18 showed a slight effect of the supernatant which was consistently observed (Figs 1 and 2). It is possible that the supernatant can confer a limited degree of cycloheximide resistance in C. cinereus but this is small compared with the effect of the ribosomes and much smaller than that reported in mammalian systems (Trakatellis et al., 1965; Felicetti et al., 1966). Any effect of the supernatant fraction was eliminated in the analysis of different strains by using a normalized assay system containing supernatant fraction from a single strain, CY18. Similarly, the results shown in Table 3 indicate the importance of using a standard concentration of ribosomes in order to compare strains and identify them as cycloheximide sensitive or resistant.

Using the standard system, the $C$. cinereus strains shown in Table 1 have been classified according to their $50 \%$ inhibitory concentrations into two groups: CY8, CY3, CY9 and CY 18 , which have cytoplasmic ribosomes sensitive to cycloheximide; and CY8.2, CY9.23 and Sp98, which have cytoplasmic ribosomes resistant to the antibiotic. The observation that $c y-2^{\mathrm{r}}$ cycloheximide-resistant mutants contain resistant ribosomes leads to the conclusion that the $c y$-2 locus codes for a ribosomal component; genetic evidence suggests that this is a protein (North, 1982).

Although the response to cycloheximide in vitro is similar to that observed in vivo (North, 1982; Traynor, 1983), the cycloheximide concentrations required for $50 \%$ inhibition of protein synthesis by cell-free extracts are several orders of magnitude higher than those required for $50 \%$ inhibition of growth of the same strain. This raises the possibility of another site of cycloheximide action in vivo. As the poly(U) system assays the elongation step of protein synthesis only, inhibition of initiation or termination may also occur in vivo (Obrig et al., 1971; Rajalakshmi, 1971) or there may be inhibition of another cellular activity. Alternatively, the high resistance levels in vitro may simply reflect the intrinsic differences between the way ribosomal activity is assayed by the two methods. 
We have shown that the $c y-2^{\mathrm{r}}$ mutation conferring growth resistance to cycloheximide in C. cinereus also causes a change in the response of the cytoplasmic ribosomes to the antibiotic. It is therefore concluded that a site on the cytoplasmic ribosomes is involved in the inhibition of polyphenylalanine synthesis in this species, the inhibition being the consequence of the formation of a stable cycloheximide-ribosome complex.

\section{REFERENCES}

BerRy, C. H. J., Ibrahim, M. A. K. \& Coddington, A. (1978). Characterization of ribosomes from drug resistant strains of Schizosaccharomyces pombe in a $\operatorname{poly}(\mathbf{U})$ directed cell-free protein synthesising system. Molecular and General Genetics 167, 217-225.

COOper, D., Banthorpe, D. V. \& Wilkie, D. (1967). Modified ribosomes conferring resistance to cycloheximide in mutants of Saccharomyces cerevisiae. Journal of Molecular Biology 26, 347-350.

Crouzet, M., Perrot, M., Nogueira, M. \& BÉGUERET, J. (1978). Genetic and biochemical analysis of cycloheximide resistance in the fungus Podospora anserina. Biochemical Genetics 16, 271286.

Felicetti, L., Colombo, B. \& Baglioni, C. (1966). Inhibition of protein synthesis in reticulocytes by antibiotics. II. The site of action of cycloheximide, streptovitacin A and pactamycin. Biochimica et biophysica acta 119, 120-129.

Hsu, K. S. (1963). The genetic basis of actidione resistance in Neurospora. Journal of General Microbiology 32, 341-347.

IbRAHIM, M. A. K. \& Coddington, A. (1976). Genetic studies on cycloheximide-resistant strains of Schizosaccharomyces pombe. Heredity 37, 179-191.

LEWIS, D. \& NoRTH, J. (1974). Linkage maps of Coprinus lagopus. In Handbook of Microbiology, vol. 4, pp. 691-699. Edited by A. I. Laskin \& H. Lechevalier. Cleveland, Ohio: CRC Press.

McEwEN, C. R. (1967). Tables for estimating sedimentation through linear concentration gradients of sucrose solution. Analytical Biochemistry 20, 114149.

NeuhÄuser, A., Klingmüller, W. \& Kandewitz, F. (1970). Selektion actidion-resistenter Mutanten bei Neurospora crassa sowie ihre genetische und biochemische Analyse. Molecular and General Genetics 106, 180-194.

Nieuwenhuysen, P., de Herdt, E. \& Clauwaert, J. (1978). Ribonucleic acid and protein content of eukaryotic ribosomes isolated from Artemia salina. Analytical Biochemistry 88, 532-538.

NORTH, J. (1982). A dominance modifier for cycloheximide resistance in Coprinus cinereus. Journal of General Microbiology 128, 2747-2753.

Obrig, T. G., Culp, W. J., McKeehan, W. L. \& HARDESTY, B. (1971). The mechanism by which cycloheximide and related glutarimide antibiotics inhibit peptide synthesis on reticulocyte ribosomes. Journal of Biological Chemistry 246, 174.

Rajalakshmi, S., Liang, H., Sarma, D. S., KisILEVSKY, R. \& FARBER, E. (1971). Cycloheximide, an inhibitor of peptide chain termination or release in liver in vivo and in vitro. Biochemical and Biophysical Research Communications 42, 259-265.

Rao, S. S. \& Grollman, A. P. (1967). Cycloheximide resistance in yeast: a property of the $60 \mathrm{~S}$ ribosomal sub-unit. Biochemical and Biophysical Research Communications 29, 694-704.

SIEGEL, M. R. \& SisLER, H. D. (1965). The site of action of cycloheximide in cells of Saccharomyces pastorianus. III. Further studies on the mechanism of action and the mechanism of resistance in Saccharomyces species. Biochimica et biophysica acta 103, 558567.

Sissons, C. H. (1974). Yeast protein synthesis. Preparation and analysis of a highly active cell-free system. Biochemical Journal 144, 131-140.

Trakatellis, A. C., Montuar, M. \& Axelrod, A. E. (1965). Effect of cycloheximide on polysomes and protein synthesis in the mouse liver. Biochemistry 4 , 2065-2071.

TRAYNOR, J. D. (1983). Resistance to protein synthesis inhibitors in Coprinus cinereus. PhD Thesis (CNAA), City of London Polytechnic.

Traynor, J. D. \& NORTH, J. (1981). Role of the cytoplasmic ribosome in cycloheximide resistance in Coprinus cinereus. Heredity 46, 287.

Wool, I. G. (1979). The structure and function of eukaryotic ribosomes. Annual Review of Biochemistry 48, 719-754. 